\title{
Gestión de la Página FORMATUR en Facebook: aportes al prestigio en internet.
}

\section{Management of the FORMATUR page on Facebook: contributions to prestige on the internet.}

MSc. Isabel Portal Díaz., ${ }^{1}$ MSc. Grisel Castellón Candelaria., ${ }^{2}$ \& MSc. Roxana Bravet Concepción. ${ }^{3}$

\section{Resumen.}

El trabajo presenta la planificación y administración de la página FORMATUR Escuela Ramal del Turismo en Facebook, a partir de la divulgación de contenidos y la interacción transparente con el público objetivo, para aumentar el prestigio de la institución en Internet. La idea surgió por la necesidad de tener presencia en internet y se ha cumplido desde enero del 2018 que se creó la página profesional, publicando diariamente noticias y eventos relacionados con el quehacer científico y metodológico de la escuela. Se incluye el social media plan, trabajo previo a la creación de la página; se divulga la planificación, gestión, actualización y estadísticas obtenidas en diferentes plazos. Los resultados de este trabajo constituyen, además, una aplicación real de que la empresa aprecie y valore el proceso de capacitación como una inversión.

Palabras claves: prestigio en internet, Facebook, planificación, gestión, actualización y estadísticas.

\begin{abstract}
.
The work presents the planning and administration of the FORMATUR School Ramal del Turismo page on Facebook, based on the disclosure of content and transparent interaction with the target audience, to increase the prestige of the institution on the Internet. The idea arose from the need to have an Internet presence and since January 2018 the professional page was created, publishing daily news and events related to the scientific and methodological work of the school. It includes the social media plan, work prior to the creation of the page; the planning, management, updating and statistics
\end{abstract}

\footnotetext{
${ }^{1}$ Escuela Ramal del Turismo, MINTUR. La Habana, Cuba. isabel@ formatur.mintur.gob.cu

${ }^{2}$ Escuela Ramal del Turismo, MINTUR. La Habana, Cuba. grisel@ formatur.mintur.gob.cu

${ }^{3}$ Escuela Ramal del Turismo, MINTUR. La Habana, Cuba. roxana@ formatur.mintur.gob.cu
} 
obtained in different terms are disclosed. The results of this work are also a real application for the company to appreciate and value the training process as an investment.

Keywords: tourism, investigation, formation.

\section{Introducción}

Las Redes Sociales se han convertido en un canal de comunicación potencial efectivo e innovador, al conectar al mundo a través de Internet. Facebook, se contextualiza en una atmósfera que tiene cambios en un ambiente globalizado, no sólo en el ámbito económico sino también en el cultural, político, educacional, entre otros, lo que debe contribuir a que las personas sean capaces de enfrentar los nuevos retos que pone la sociedad.

El presente trabajo surge por la necesidad de tener presencia en internet, socializando o divulgando contenidos e interactuando de manera transparente con el público objetivo, para aumentar el prestigio de la institución en los medios digitales.

En la actualidad el ecosistema de los medios está cambiando significativamente, han aparecido maneras innovadoras y alternativas de comunicación dentro de las cuales se encuentran las redes sociales. Las nuevas tecnologías están teniendo un auge vertiginoso y están impactando de manera significativa algunas de las formas de relación entre las personas.

Facebook ha marcado un antes y un después en cuanto a comunicación digital, ya que ha sido no la primera, pero sí, la más fuerte en cuanto a volumen de las redes sociales y ha conseguido cumplir la misión empresarial que su creador Mark Zuckerberg, declarara en el 2004: "Hacer que las personas puedan compartir y hacer del mundo un lugar más abierto y conectado". A partir de 2017, el nuevo lema para la compañía, es "ofrecer a la gente el poder de construir comunidades y hacer que el mundo esté más unido".

La red social Facebook proporciona una mayor capacidad de acción, porque se hace cómplice de la necesidad adolescente de identificación con un contexto y estilo de vida. Se destaca la importancia de esta red social como herramienta que podría tener un potencial no explotado en su máxima capacidad como posible plataforma de participación por medio de la cual las personas expongan sus ideas y propicien discusiones que fomenten la cultura participativa.

Está muy lejos de ser un sistema perfecto, pero sin duda es una de las herramientas más sencillas para los usuarios menos tecnificados, y de ahí su gran acogida y adopción en masa, especialmente en Latinoamérica. En el aspecto profesional también supone un importante apoyo, y en el caso de las empresas, Facebook es de hecho la red social que más visitas lleva a sitios web y blog, aunque han surgido otras redes sociales que están haciendo gran competencia.

Es muy conveniente para construir la imagen digital, especialmente desde el punto de vista profesional, es por ello que fue seleccionada en un primer momento para tener presencia en las redes sociales.

Un error muy común que cometen las empresas es estar en redes sociales simplemente por estar. Gestionar de manera profesional la presencia de una empresa en Facebook, requiere capacitación en diversas temáticas, y es por eso que, en 2017, luego de 
transcurrido casi un año, un grupo de profesores de FORMATUR Escuela Ramal del Turismo, se gradúa del primer curso del país, de Community Manager Hotelero, impartido por prestigiosos profesionales de la Cadena Blue Diamond Resorts.

En el propio transcurso del curso, se fue concibiendo la idea de la creación de la página profesional del centro, y en enero del 2018 se materializa, publicando desde entonces, y diariamente, noticias y eventos relacionados con el quehacer científico y metodológico de la escuela, de acuerdo con la planificación y ejecución de las actividades.

El informe presenta la planificación, gestión y principales aportes de la página FORMATUR Escuela Ramal del Turismo en Facebook, a partir de la divulgación de contenidos y la interacción transparente con el público objetivo.

\section{Métodos:}

1. Social Media Plan (SMP). Paso previo a la definición del producto.

El primer paso antes de la creación de la página lo constituyó el SMP, es un documento en el que se detallan las estrategias de redes sociales que se van a llevar a cabo para alcanzar los objetivos de marketing de la empresa o el paso previo a la definición del producto. Para ello, se respondieron interrogantes, que permitieron definir quiénes somos y qué se quería mostrar, las principales ventajas y diferencias de la competencia, quiénes son y qué contenidos ofrecen.

Los pasos seguidos en la definición del SMP son los siguientes:

$1^{\circ}$ Paso $\rightarrow$ Objetivos: ¿Qué se quiere conseguir?

$2^{\circ}$ Paso $\rightarrow$ Estrategias: ¿Qué camino se va a tomar?

$3^{\circ}$ Paso $\rightarrow$ Acciones: ¿Cómo se va a conseguir?

$4^{\mathrm{o}}$ Paso $\rightarrow$ Elección de los canales: ¿Dónde se va a actuar?

$5^{\circ}$ Paso $\rightarrow$ Planificación específica.

$6^{\circ}$ Paso $\rightarrow$ Establecimiento y medición.

$7^{\circ}$ Paso $\rightarrow$ Elección de herramientas a utilizar para la gestión y la medición.

$8^{\circ}$ Paso $\rightarrow$ Elección del Personal necesario.

$9^{\circ}$ Paso $\rightarrow$ Protocolo de actuación para casos de crisis de reputación.

$10^{\circ}$ Paso $\rightarrow$ Lista de medios y presupuesto si fuera necesario.

A continuación, se mencionan los principales elementos del SMP concebido por las autoras del trabajo.

Principales debilidades, amenazas, fortalezas y oportunidades, así como, los valores compartidos y deseados:

D: Insuficiente presencia en internet y no hay posicionamiento web.

A: Pérdida de la privacidad o su violación y la competencia de otras escuelas ramales o centros de capacitación del país dentro o fuera del MINTUR y escuelas de hotelería del mundo.

F: Marca FORMATUR reconocida internacionalmente, por la calidad del modelo cubano de formación y capacitación, con liderazgo y prestigio. 
O: Socializar la información, tener presencia internacional en las redes sociales y ser un canal de comunicación con el público objetivo.

\section{Valores:}

Valores compartidos

Profesionalidad

Comportamiento ético

Valores deseados

Trabajo en equipo

Creatividad

Sentido de pertenencia

\section{Objetivo:}

Inicial: Aumentar el prestigio de la institución a partir de la divulgación de contenidos y la interacción transparente con el público objetivo.

Más adelante: "Aumentar el número de visitas a la Web desde Facebook durante el año".

Estrategia: Captación de seguidores a partir de diferentes tipos de publicaciones que brinden información de la marca.

Acciones generales: Ofrecer contenido interesante y exclusivo.

Acciones específicas: Publicar diariamente noticias y eventos relacionados con el quehacer científico y metodológico de la escuela de acuerdo con la planificación y ejecución de las actividades.

Tiempo de actuación: Largo plazo.

Equipo de trabajo: Son los encargados de la planificación y administración de la página: Directivos y metodólogos: Directora, subdirectores, metodólogos y jefes de departamentos.

Community Manager (CM): MSc. Isabel Portal Díaz.

Reservas CM: MSc. Grisel Castellón Candelaria y MSc. Roxana Bravet Concepción Imágenes: Fotógrafo, "cámara fotográfica” y diseñador.

Traducción e interpretación: inglés: Aleida Vega; francés: Gilberto G. Rose.

\section{Definición del producto: FORMATUR Escuela Ramal del turismo.}

Objetivo: Promocionar noticias y eventos relacionados con el quehacer científico y metodológico de la escuela.

Eslogan: "No solo preparamos a los directivos y reservas del sector turístico, implicamos al claustro en el desarrollo del turismo y en la dirección metodológica de los Centros de Capacitación." 
Imagen N. 1 Botones call to action.

\section{Perfil}

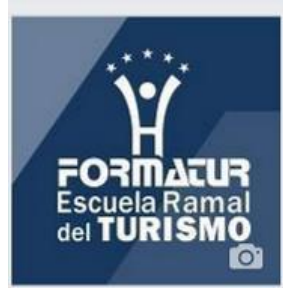

Formatur Escuela Ramal del turismo @formaturescuelaramal Inicio

\section{Portada}

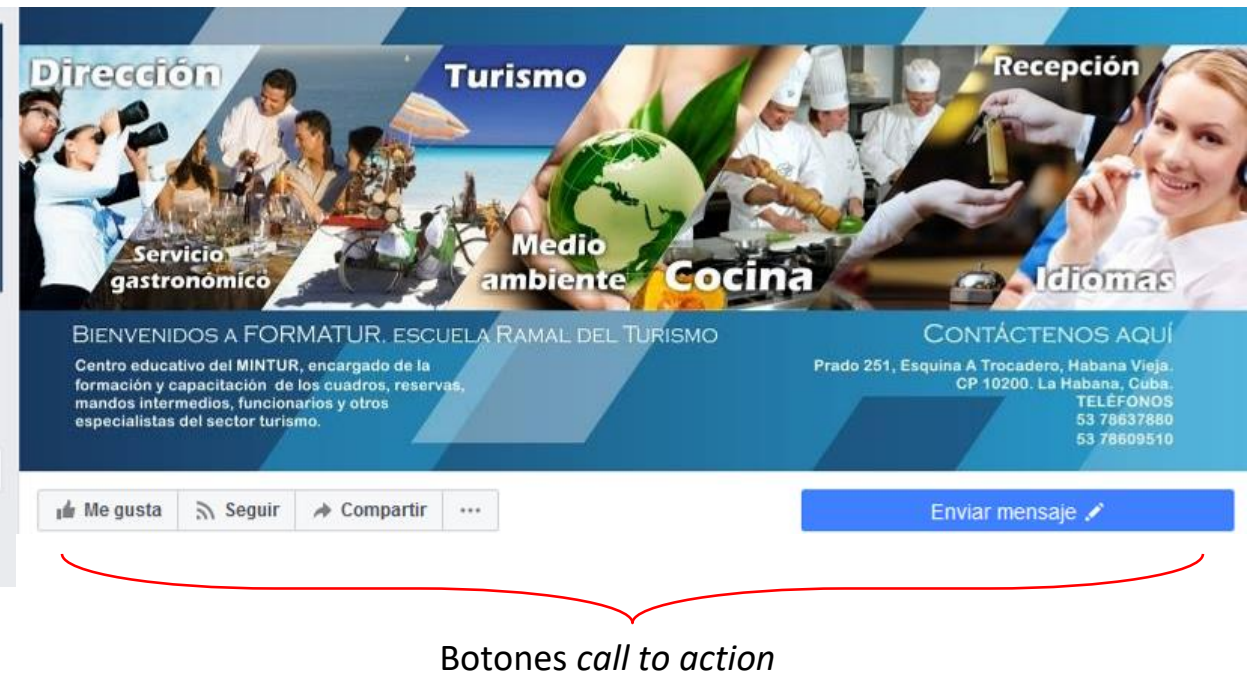

Nombre de la página: Acompañado de una descripción que incluye un call to action ${ }^{4}$ para visitar la página web, incluya información: @ formaturescuelaramal

Tiempo de respuesta: Normalmente se responde en 2 días (teniendo en cuenta que el fin de semana no se puede acceder a la página)

Información de contacto adicional

Vínculo sitio web corporativo: Pendiente hasta que se publique el sitio web de la escuela que se encuentra en construcción.

\section{Plan de crisis o protocolo de actuación.}

Las crisis en Redes Sociales son una realidad y es un riesgo al que se enfrenta hoy en día todo el que tenga presencia en Internet. Las autoras elaboraron el plan de Crisis para asentar las bases sobre cómo actuar ante una situación de riesgo para la reputación online del centro.

\section{Acciones internas para mejorar la gestión de la página y su posicionamiento} web.

Con el fin de que el colectivo laboral apoye la interacción con la página de la escuela, se han circulado correos electrónicos incitando a los profesores a seguirnos, con el Asunto: SÍGUENOS EN FACEBOOK. De esta manera se ha logrado que algunos puedan interactuar y participar, apoyando a la escuela y a las administradoras en una labor extra a su contenido de trabajo. También se ha incitado a los estudiantes, a los participantes en eventos a acceder a la página para que nos sigan y compartan las publicaciones en las que ellos aparecen y recomienden la página a otros.

\section{Otras acciones realizadas para mejorar el posicionamiento web. Seguimiento a grupos y páginas.}

\footnotetext{
4 También llamado "Ilamada a la acción". Es un botón o enlace situado en el sitio o página que busca atraer clientes potenciales y convertirles en clientes finales
} 
Para aumentar la visibilidad de la página, se han compartido publicaciones de otras páginas de Centros de Capacitación del turismo, instituciones turísticas, se han utilizado a los directivos, trabajadores y cursistas en el centro como embajadores de la marca. También se etiquetan a los directivos de la escuela que participan en eventos de relevancia relacionados con el sector turístico, se promociona las visitas de importantes personalidades del mundo de la hotelería y el turismo en el centro, a prestigiosos chefs, artistas, entre otros; tanto nacionales como de otros países que han interactuado con el colectivo en actividades docentes y científicas.

Otra estrategia que nos da mayor visibilidad ha sido la de seguir e indicar "Me gusta" como página, a páginas profesionales dentro del sector turístico de nuestro país y del mundo, comenzando por la del Ministerio del Turismo de Cuba, las OSDES y empresas hoteleras y extrahoteleras, los centros de capacitación del país, etc. También se siguen grupos y nos comparamos con las páginas de los centros de capacitación del país, en cuanto a cantidad de seguidores, interacciones y reacciones.

Utilización de hashtag: Propio: \#Formatur. Otros: \#Cuba, \#CocinaCubana, \#FITCuba2018, \#SeminarioGourmet \#Excelencias20 \#ExcelenciasGourmet \#TapasyCoctelesenLaHabana. Se realiza rastreo de hashtag para incluirlos en las publicaciones.

\section{Publicaciones: Estructura y tipología}

Como se ha explicado anteriormente todas las publicaciones que se realizan en la página están relacionadas con el quehacer docente y científico de la escuela; pero a su vez pertenecen a subcategorías dentro de esta clasificación. Por ejemplo, en algunos casos, son derivadas de acciones de colaboración internacional, otras de la presencia de FORMATUR en eventos de relevancia a nivel nacional, algunas asociadas a la labor encomendada al centro en cuanto a la asesoría metodológica a los Centros de Capacitación del Turismo existentes en las delegaciones territoriales del país. Se publican también actividades asociadas a los valores declarados para el centro, como son los actos realizados por determinadas efemérides, o el reconocimiento que se dio a todos-incluidos los trabajadores del turismo-, que brindaron su apoyo y solidaridad con los familiares de las víctimas del accidente aéreo ocurrido el 18 de mayo de 2018.

La community manager y su equipo de administradoras, en correspondencia con la capacitación recibida y las tendencias que cada día se renuevan, han utilizado los post siguientes:

- $\quad$ Producto (Todos con fotos propias)

- Videos propios

- $\quad$ Video con Link

- Compartir de otra página

- Álbum

- $\quad$ Programados para otra fecha 


\section{Resultados}

\section{Estadísticas y métricas.}

Las redes sociales cuentan con herramientas que ayudan a medir los resultados finales de las estrategias, y por supuesto Facebook ofrece una información muy interesante, que es gratis, en cuanto a cómo le fue a la página. Existen otras, pero hay que pagarlas, como, por ejemplo, Facebook Insights para evaluar el desempeño que ha tenido la página.

A continuación, se ofrece un resumen de las estadísticas generales de la página:

- El alcance ha sido bueno, llegando a más de 1300 personas (dato que crece a cada segundo)

- La interacción de los visitantes con las publicaciones es buena.

- Se ha obtenido un $100 \%$ de índice de respuesta a los mensajes de los visitantes, que puede considerarse como muy bueno, con un tiempo medio de repuesta de minutos (datos que crecen a cada segundo)

- Se han publicado videos que despertaron interés en verlos, ya que fueron reproducidos al $100 \%$.

- Las publicaciones e interacciones tuvieron buena aceptación en el público, no se le ha realizado ningún comentario negativo. La mayor reacción que existe es "Me gusta"

Resumen de enero a septiembre de 2018: Seguidores $\underline{44}$ y Reacciones $\underline{427}$ "Me gusta" (dato que crece a cada segundo)

\section{Análisis técnico económico.}

Facebook es un sitio gratuito y según ellos nunca se pedirá pagar por seguir usándolo, pero para llegar a más personas sí cobran por promocionar el negocio, cosa que no ha sido necesaria, por tanto, los seguidores de la página son todos orgánicos ${ }^{5}$. Solo se ha consumido la electricidad de la PC, los servicios de la red, el salario por alrededor de 3 horas diarias que la community dedica a este trabajo, así como el alrededor de 4 horas semanales que las otras 2 administradoras de reserva le dedican.

\section{Discusión}

\section{Aporte social:}

Es una poderosa herramienta innovadora de interacción en una plataforma de medios de comunicación social digital, que potencia el reconocimiento y fidelidad de la marca FORMATUR a nivel nacional e internacional, además de que propicia un mayor impacto en la comunidad de seguidores.

La práctica en los medios sociales digitales ha permitido que los colectivos de profesoras se preparen para la formación y capacitación de directivos y Community managuer de las OSDES y empresas del turismo, por lo que se ha convocado a la segunda edición del curso Community managuer a comenzar en el mes de octubre.

${ }^{5}$ Seguidor orgánico se denomina a la persona que ve alguna publicación no pagada procedente de la página. 
Más adelante se ampliarán los perfiles profesionales del centro en las redes sociales, como LinkedIn, que es una red para profesionales donde además de darse a conocer el centro, se pueden hacer alianzas y establecer relaciones con otras escuelas o profesionales con actividades afines a la escuela.

Debemos mejorar la calidad en la obtención de las imágenes, dado que se está haciendo con cámaras de teléfonos de gama media, los cuales no aportan la calidad que se requiere.

Continuaremos Innovando en cuanto a creatividad porque las redes sociales evolucionan constantemente, por lo que los contenidos deben ser cada vez más interesantes y atractivos.

Debemos crear un grupo cuando crezca la comunidad, porque son una herramienta que nos ayudará, son un canal adicional para acercarnos a nuestro público de una manera distinta, aumentando la relación con ellos. Si son usados de la manera correcta, pueden brindarnos muchos beneficios.

\section{Conclusiones.}

> Como base fundamental para poder posicionar en la web a FORMATUR Escuela Ramal del Turismo, un equipo de profesores recibió la capacitación necesaria para elaborar el social media plan, trabajo previo a la creación de una página profesional en las redes sociales; y posteriormente llevar a cabo su administración.

$>$ La página profesional "FORMATUR Escuela Ramal del turismo" publicada en Facebook desde enero de 2018, ha permitido divulgar noticias y eventos relacionados con el quehacer científico y metodológico de la escuela, interactuar de manera transparente con el público objetivo, y por tanto lograr un posicionamiento en la web.

$>$ En este trabajo se socializa cómo se planifica y administra una página profesional de un centro educativo, y el necesario análisis que debe hacerse de las estadísticas para evaluar si se cumple su objetivo, y en qué aspectos debe mejorarse.

\section{Referencias Bibliográficas}

Aránzazu M, Alonso S, Arcos S. Turismo 2.0: Cómo adaptarse a la Revolución Digital.2013.

Compendio de materiales del curso Community Manager de FORMATUR Escuela Ramal del Turismo y la cadena BlueDiamond Resort. La Habana, 2017

Comprender y analizar las estadísticas de una página de Facebook. Disponible en: http://es.cm.net/faq/10077-comprender-y-analizar-las-estadisticas-de-unapagina-de-facebook. 
Empleados como embajadores de marca. Puro Marketing. Consultado abril de 2018. Disponible en: http://www.puromarketing.com/14/26725/empleados-comoembajadores-marca-beneficios-reporta-empresas.html

Embajadores de marca y su importancia. Puro Marketing. Disponible en: http://www.puromarketing.com/42/18836/importancia-gran-poderembajadores-marca.html

Guía completa de informes de analítica de Facebook para páginas de fans. Disponible en: http://dalealaweb.com/2016/03/guia-analitica-fanaticas-paginas-fans Consultado en Septiembre de 2017.

La figura del Community manager Disponible en: www.puromarketing.com/42/12186/figura-community-managerimprescindible-como-propio-gerente.html

Lluveres C. Guía completa de informes analítica nativa de Facebook, para páginas de fans. 2017.

Pazmiño PA. El impacto de las redes sociales y el internet en la formación de los jóvenes de la Universidad Politécnica Salesiana: Caso carrera de Comunicación Social Sede Quito. Tesis previa a la obtención del Título de: Licenciada en comunicación social. 2017.

Vilallonga A. Imagen personal y corporativa. Disponible en: http://www.andreavilallonga.com 


\section{Para citar el artículo indexado.}

Portal I., Castellón G. \& Bravet R. (2018). Gestión de la Página FORMATUR en Facebook: aportes al prestigio en internet. Revista electrónica Explorador Digital 2(2), 26-35. Recuperado desde:

http://cienciadigital.org/revistacienciadigital2/index.php/exploradordigital/article/view/330/7 $\underline{42}$

\section{Ciencia \\ Digital \\ Editorial}

El artículo que se publica es de exclusiva responsabilidad de los autores y no necesariamente reflejan el pensamiento de la Revista Explorador Digital.

El articulo queda en propiedad de la revista y, por tanto, su publicación parcial y/o total en otro medio tiene que ser autorizado por el director o editor de la Revista Explorador Digital.
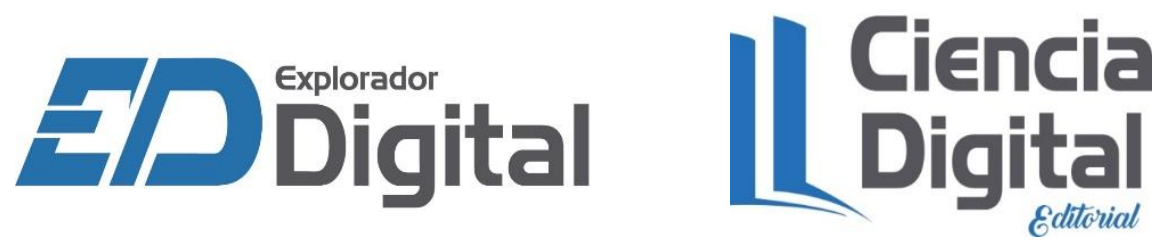